\title{
Conceptions of Importing Talents Overseas for China's Colleges and Universities in China's New Normal
}

\author{
Dan WANG \\ Human Resources Construction Office, Dalian University, Dalian 116000, China \\ *Emial: 714497987@qq.com
}

Keywords: China's new normal, Colleges, Universities, Importing, Talents overseas.

\begin{abstract}
In recently years, China's economy has turned into a development stage overlaid within a period of gear shifting for speed increasing, a period of labor pains for structure adjustment and a period of digestion for previous policies, which formed an economy status of "Intermediate Speed, Optimized Structure, New Impetus and Multiple Challenges" and it is called "China's New Normal". In China's New Normal, new attitude is necessary. With the international competition getting more and more intense and innovation driving becoming more and more urgent, the human resources career in China has got a new start point in China's New Normal. As an important puzzle of the innovation driving development strategy, the importing of talents overseas for China's colleges and universities is significant to the improvement of national competitiveness and promoting of innovation and development. The importance goes without saying. "Advancing with the times", the human resources career in China's colleges and universities has to be innovated and the "New Normal" of human resources has to be constructed to get ready with the adjustment of the "New Normal" of China's economy nowadays.

In recently years, China's economy has turned into a development stage called "China's New Normal" and faces unprecedented chances and challenges. The China Communist Party Central Committee has ordered clearly that "Adapt the New Normal, Hold the New Normal and Lead the New Normal" and impel the economy to transit and update. During "China's New Normal", the human resources are the main power of development. As an important intelligence support of the economy transition and updating, the human resources career shall consider in a certain aspect of the new structure, new development and new power of the "New Normal" to innovate the human resources conceptions. Since the 18th National Representative Plenary Session of China Communist Party, Chairman XI Jin-ping has paid high attention to the importing of talents overseas. In political aspects, Chairman XI Jin-ping replaces this career as a puzzle of realizing the "Dual-Century Attemptation Goal" and realizing the "Chinese Dream" of the Great Revival to consider, plan and impel. Nowadays, Chairman Xi Jin-ping "sends far and wide to invite men of ability" to put into effect the strategy of importing talents overseas and blows out the Assembly of recruiting from China to the whole world.
\end{abstract}

\section{The Status in Quo and Challenges of Importing Talents Overseas for China's Colleges and Universities in "New Normal"}

Theodore W. Schultz (1902-1998), an economist from America who won the 1979's Nobel Economy Prize, said "Human capital contributes to labor productivity and to entrepreneurial ability". The prestige and grade actually depend on the abilities of tuition and scientific research. To improve these abilities, it is not only depending on the front-line lecturers and scientific research personnel, but also depending on importing the famous scholars and high-class experts who hold the international pioneering scientific research technologies, hold the ability of constantly exploiting complexions and have obtained prominent achievements. Hence, importing talents overseas and building up a professional, high-quality, rationally-structured and constantly innovatability holding teachers' team are the guarantee to keep colleges and universities moving to high class.

For years, the career of importing talents overseas for China's colleges and universities has been 
effectively processed. "A university is not constructed by buildings, it is constructed by masters; only a university which has first-class masters can be called a first-class university", this is a prevalent conception of high-class talents among China's colleges and universities. However, there is a pearl of wisdom in Chinese Language that is "It takes a decade to cultivate a tree; it takes a century to cultivate a people". The human resources cultivation is a long-term system project. While the persons with ability are eager to meet the needs of masses, it could be a shortcut solution to import talents overseas.

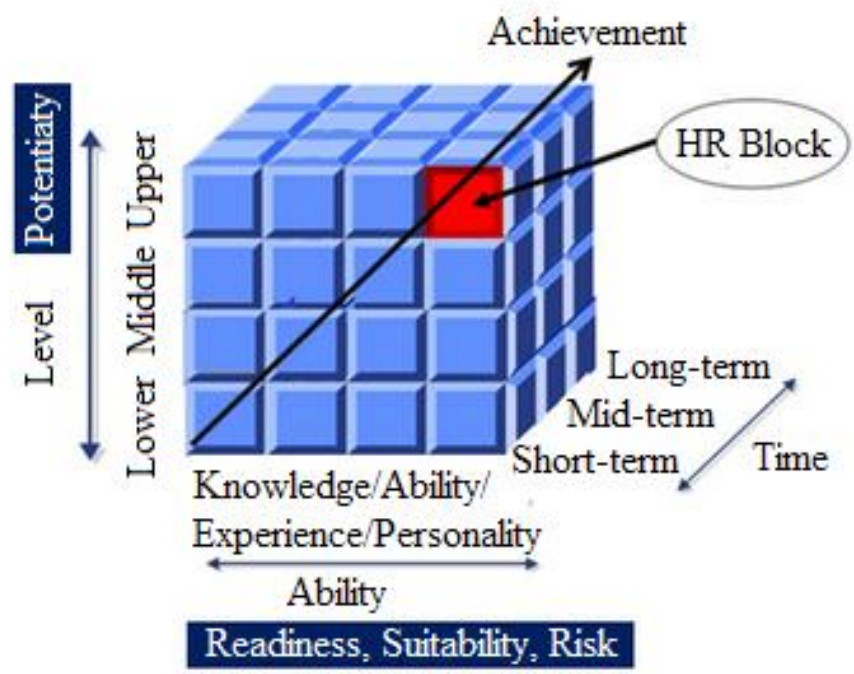

Figure 1 Human Resources Standard Model.

Shown in Fig. 1 is a modeling figure to explain the Achievement and Ability of human resources according to "The Theory of Contribution" of Peter F. Drucker (1909-2005), in which the "Human Resources" is not only representing the quantity, but also showing the key ability that is needed in enterprises' strategies implementing.

In recently years, the career of importing talents overseas in China has been effectively processed. Hundreds of excellent persons with ability have settled down in China and successfully obtained brilliant achievements in their colleges and universities. In "China's New Normal", demands far exceed supplies in human resources field. Since the unoptimistic status in quo of human resources development, "China's New Normal" has made a new challenge to the career of importing talents overseas at the same time.

The scale of importing talents overseas is too small at present and shall be optimized. The scale of importing talents overseas cannot meet the need of national economy development strategies. The impetus of importing talents overseas in high and new technologies field is insufficient and the quantity of importing talents overseas in tuition and scientific research field is not enough. The human resources importing structure shall be optimized, which means that the ratio of talents in high and new technologies field is low and the impetus of directly importing talents overseas shall be enhanced. Firstly, the ratio of high academic qualified talents is low in China at present. The indigenous world-class scientists who won international authoritative science prize in China are very rare. According to the rank of the quantity of foreign nationality academicians in international authoritative science academies, China is not also lower than high-class developed countries, but also lower than some mid-class developed countries. Secondly, the innovation-type talents are in shortage. TIAN Li-pu, the former director general of National Intellectual Property Department in China, has mentioned in his book "Self-Dependent Innovation and Intellectual Property" that: The patents in China are mainly for food and other civil use, since the patents in developed countries are mainly for high-tech fields. In the terms of the quantity of scientists' high gold-contented patents, China is still much lower than developed countries. Thirdly, the talents in China are geographically unbalanced developed. Talents with ability are mostly living in the south-east coastal cities. Fourthly, the ratio of high-class talents in China's colleges and universities is still low. Internationally discipline-leading, 
high-class and high academically qualified talents are urgently needed at present, to inject international advanced technologies for the career of higher education in China.

The management structure of importing talents overseas is lagged at present and shall be innovated. Firstly, it is the problem of the management structure. The processes of importing talents overseas have to be declared to too many government departments with complicated procedures which may lead to difficulties of coordination, low efficiency or inappropriate services. Secondly, it is the problem of the marketization structure. The main function of the employers and the decisive effect of the market deposition for talents overseas have not been given to full play. The participation of Human Resources secondary agents and the utilization efficiency of market resources have been insufficient. The working procedures of importing talents impelled and implemented by China's government have been inertial and it has caused some problems of importing talents indexation, projects fragmentization, competition homogenization, etc. Thirdly, it is the problem of incentive and restriction mechanism. On the aspect of estimation and incentive mechanism, since some colleges and universities ignored the characteristics and requests of the talents overseas, it is not good to mobilize their enthusiasm and innovations and there are some kind of phenomenon called "Imported but not Stay" at the same time. Besides, the restriction and supervision mechanism shall be optimized and the restriction and withdraw mechanism shall be intensified.

The legislation management of importing talents overseas is lagged at present and the policies management shall be optimized. Firstly, it is the problem of legislation in China. In the recent decades, a talents scramble war among the countries all around the world has begun and importing talents overseas has been a strategic issue. On the aspect of legislation implementation in different countries, it is mainly about the Nationality Law, Immigration Law and the legislation for the reflux of talents overseas. It is also an unavoidable issue of legislation for importing talents overseas in China. Secondly, it is the problem of policies management. Problems like policies fragmentization, policies gaining channel blockage, policies incentive period-shortenization, overall planning and long-term planning lacking, etc. are existing in the management service for the talents overseas. Thirdly, it is the problem of social security policies. The policies for the convenience of Entry-Exit and Residence for High-class Talents shall be optimized. Especially on the aspects of social security, medication, insurance, education, family resettlement, etc., the policies and legislations that the talents overseas actually need shall be optimized.

\section{Initiatives of The Innovation and Transformation Development of Importing Talents Overseas for China's Colleges and Universities in China's New Normal}

"National Program for Medium and Long-term Talent Development (2010-2020)" proposed that, there should be five principles, namely, giving priority to development, taking the cultivation of people as the starting point, carrying out reform and innovation, promoting equity, and improving quality. These five principles mainly introduced the strategic positioning, strategic priorities and major tasks of talents development. To develop the career of importing talents overseas for China's colleges and universities in China's New Normal, China shall follow the national development strategy goals, be directed with the "Importing Higher-class Talents Overseas Plan" (also known as "Thousand Talents Plan") and import strategy scientists and discipline-leading talents who have the abilities of breaking through in key technologies, developing high-tech industries and emerging new disciplines to settle down in China, for the national key innovative projects, disciplines, laboratories and central enterprises, state-owned commercial financial institutions, high-tech industrial development zones, etc. With the development of the National Program for Medium and Long-term Talent Development, China's colleges and universities shall aim the economic and social development targets and the problems of human resources development of its own laws, innovate the human resources conceptions, import excellent talents, stimulate the talents' abilities and replace the major issue "Import Excellent Talents, Develop Higher Education Career" at the first position. 


\section{Enhancing the Top Design and Accelerating the System Construction}

Make the Management System of Importing Talents Overseas Smooth. From learning the experiences of the developed countries, the management system of importing talents overseas in China shall be optimized and reconstructed. Management responsibilities and public resources shall be integrated and departments' responsibilities relations shall be rationalized to promote the establishment of a responsible, efficient functioning and legal guaranteed management system of importing talents overseas.

Shown in Fig. 2 is is a figure to explain the management of talents overseas integration.

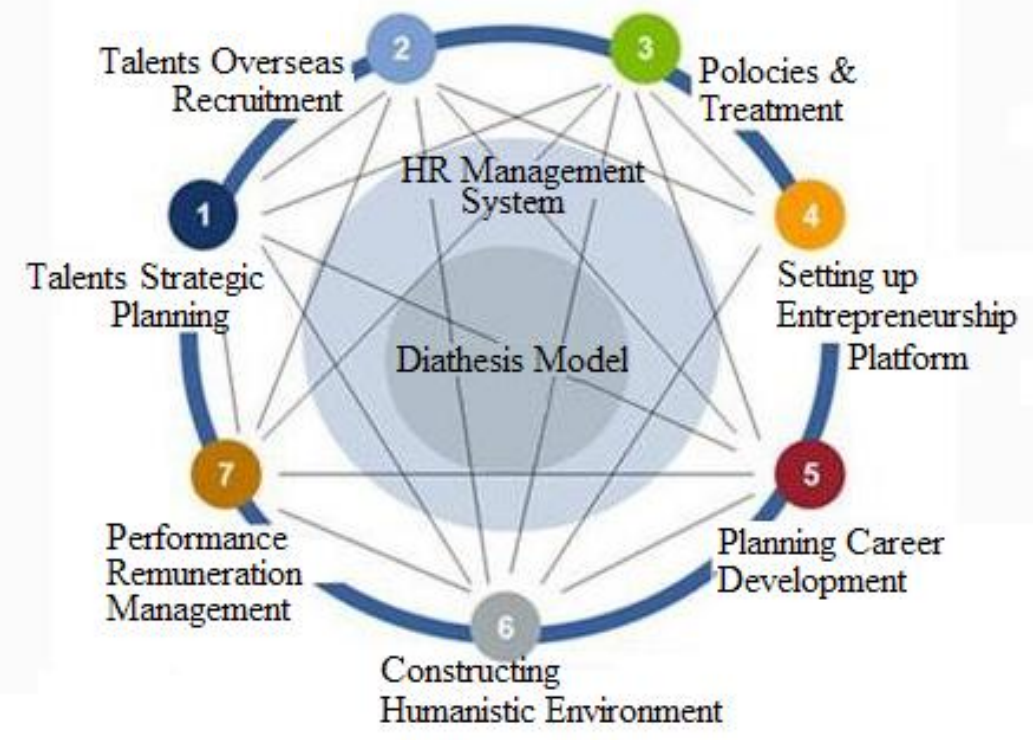

Figure 2 Management of Talents Overseas Integration.

Enhance the Legal Construction of Importing Talents Overseas.

A talent access permission system with uniform standard, standardized procedures, transparency, efficiency shall be constructed soon to build a connection with the talents importing system; Enhance the research of policies from other countries and learn the experiences of labor market testing, integral assessment and immigration quotas in developed countries to explore the skilled immigrants legislation system in compliance with China's national conditions, resolve "the Last Mile" problem of law enforcement and policies implementation and ensure the various policies and regulations of importing talents overseas comprehensively implemented.

\section{Deepening the Reform of Mechanism and Promoting the Mechanism Innovation}

Restore the Marketization of Importing Talents Overseas.

Fully utilize the decisive role of market of importing talents overseas, build up the human resources supply \& demand mechanism, the price mechanism and competition mechanism, lead the positive competition of importing talents among the universities and colleges in China, achieve the goal of win-win cooperation and stimulate social forces participating in professional service organizations for talents overseas to build up a working mechanism of competition, cooperation, fusion, intercommunication and mutual learning for talents domestic and overseas.

Optimize the Incentive and Restriction Mechanism.

Build up the incentive mechanism for the basic scientific researching talents overseas in the way of reformation issues achievements assessment and emphasize the decisive role of innovation in the achievements assessment; Optimize the incentive mechanism of income distribution and intellectual property rights for the innovative talents and endow these talents with the remunerations of Patent \& Achievement Benefits Citizens to ensure the lawful income channels of cashing out in capital market; Optimize the recognition awards system of "Friendship Award" for the talents overseas to improve the sense of honor. At the same time, restore the restriction and supervision mechanism for the talents overseas, enhance the restrictions of contracts and laws and strengthen the entry reviewing and elimination mechanism to prevent the risks of importing. 
Enhance the Service and Security Mechanism.

Build up the "Green Passage" information sharing mechanism for the talents overseas to optimize the procedure of importing, simplify the relevant procedures, optimize the "One-Station" service mechanism and set up a more convenient and sufficient management and service system for the talents overseas. On the aspects of social security, medication, insurance, education, family resettlement, etc., an "Internationalization Window" shall be added in the system design to build up a more convenient and sufficient "Green Passage" for the talents overseas and their families.

\section{Optimizing the Service Conceptions and Creating a Green Environment Fast}

Enhance the Strategies Implementation and Create the Atmosphere of "Policies Attract Talents".

In recent years, provinces and cities in China unveiled kinds of provisional regulations, implementing regulations and policies of importing talents, especially including preferential policies of social security, projects funding support, welfare benefits, human resources agencies and employment migration for the talents overseas. However, the policy "Higher-class \& Highly-skilled Urgently Needed Talents Green Passage" shall be promulgated sooner to resolve the problems of housing, spousal employment, schooling of children, academic communication funding, innovation and entrepreneurship support, immigration convenience, etc. for the talents overseas setting up in China. Similarly, the preferential policies of title promotion and remuneration shall be also promulgated to prevent the worries of the career planning of the talents.

Enhance the Incentive Mechanism and Create the Atmosphere of "Careers Attract Talents".

Build up the career platforms, innovate the modes of attracting and recruiting, enhance the incentive mechanism for the higher-class talents and construct the systems of Human Resource Development Special Funds, Higher-class Talents Special Allowance, etc. Utilize the modes of work-team importing, core talent driving importing and high-tech project development importing etc., build up the importing mechanism in compliance with the characteristics of talents overseas and rely the scientific research positions of the emphasis labs and the academic leaders of emphasis research institutions to implement the open recruitment all over the world. Focus on the importing of excellent young talents overseas. The outstanding talents can be directly appointed on leadership positions and corresponding senior professional and technical positions, to cultivate a team of higher-class talents overseas with strategic visions and leading abilities in professional field and a team of high-end leading talents.

Enhance the Awareness of Service and Create the Atmosphere of "Affections Attract Talents".

Actively adapt the New Normal of human resources. The key of importing talents overseas is intentions, concerning and the precise positioning of the importance of importing talents overseas. Establish the conception of "Talents are the First Resources" in the human resources conceptions. Without talents, the education development would be like "A Water without a Source, A Tree without Root"; Establish the conception of Humanism in the human resources management and create the atmosphere of "Hold people's hearts" and "Harmonious and Progressive"; Establish the conception of "Talents are the Wealth, Talents are the Benefits, Talents are the Competitiveness and Talents are the Development Potentials" in the human resources career. Implement the "Train Service System" for the talents overseas. High quality service is the guarantee of creating excellent talents development environment. In China's New Normal, it is necessary not only recruiting the talents but also retaining the talents, to make the talents feel a sense of belonging, settle down, bloom and bear fruits.

Enhance the Policies of Security and Create the Atmosphere of "Remunerations Attract Talents". Fully understand the actual needs of the talents overseas, optimize and formulate the practicable policies of preferential treatment, support high quality living conditions and laboratory conditions and offer higher working and living conditions than other similar personnel on the aspects of household allowance, research funding, remunerations, logistical support, welfare benefits, etc. At same time, policies with continuity and feasibility shall be promulgated to create all conditions for the urgently needed talents overseas with flexible methods.

Enhance the Humanities Guarantee and Create the Atmosphere of "Environment Attracts Talents".

To ease the status of intense work for the talents overseas after settled down in China, relatively 
relaxed working environment shall be offered to help them adapt the research and management mechanisms in China, support them to organize academic conferences, help them be recognized in a larger range and assist them to plan development directions in the future. At same time, actively support them declare national major honorary titles, publicize and popularize their research results, advanced deeds and dedications and create a good atmosphere of respecting knowledge and respecting talents. The talents overseas shall fully have the rights of research independence, human resources management and funding domination in their teaching and research works. Relaxed academic environment and a good atmosphere for cooperation shall be created to inspire them to innovate and seeking differences, to fully get involved in the first line of teaching and researching. Import excellent talents who are in compliance with transformation development, green development and innovation development and ensure the talents overseas to adapt the "New Normal" through a series of incentive measures.

\section{Conclusions}

Importing talents is a systematization project, which involves various aspects of economics, politics, culture, environment, services, etc. Uphold the conceptions of "Environment gathers Talents, Talents lead the Development", optimize the service conceptions, recruit talents from everywhere, with various of abilities, build up a platform system of various of channels, grades and forms for various of talents to assist the talents overseas "Well Imported, Well Settled, Well Worked" after settled down in China. In China's New Normal, universities and colleges shall import excellent talents overseas with pragmatics, attentiveness and innovations to adapt the economic development in China's New Normal and to meet the requirements of Chinese higher education endowed by this era.

\section{References}

[1] J.P. Xi, Talents are the First Resources, "XI Jin-ping's Discusses Excerpts about Science \& Technology Innovations" (Volume VIII) Firmly Grasp the Major Initiatives of Talents' Attraction, Central Archives of the CPC Central Committee, Beijing, 2016.

[2] Forwarded by General Office of the Communist Party of China, Comments of Implementing Plans for Importing Higher-class Talents Overseas by Talents Coordination Group of the CPC Central Committee, "Importing Higher-class Talents Overseas Plan (also known as 'Thousand Talents Plan')”, Beijing, 2008.

[3] Ministry of Education of the PRC, "Comments of Further Strengthening the Importing of Outstanding Talents Overseas by Ministry of Education of the PRC", Ministry of Education of the PRC, Beijing, 2007.

[4] Strategic Theory Research Office of CPC Central Committee, Be well prepared with Importing Talents Overseas in China's New Normal, "China Organization and Personnel News", Beijing, 2015.

[5] Z.H. Sun, Be well prepared with the conceptions of Importing Talents, “Top HR”, Beijing, 2014.

[6] X.F. Li, Human Resources Management will be the New Normal of Enterprises in China, "Hubei Dailey”, Wuhan, 2015.

[7] R.H. Shen, The Innovation Conceptions of Human Resources in China's New Normal, "Wenhui Bao", Shanghai, 2014.

[8] L.G. Song, How to be well prepared with the Human Resources Career in China's New Normal, “CHINA QI”, Beijing, 2016.

[9] H.Y. Wang, Four Disparities Obstructing Higher-class Talents Returning back to China, "Xinhua Net", "China Youth Daily”, Beijing, 2013. 